\title{
A NEW METRIC FOR A METRIC SPACE
}

\section{ROBERT B. FRASER}

1. Introduction. Bing has shown [1] that there is always a convex metric (in the sense of Menger [6]) for a Peano continuum, uniformly equivalent to the original metric. His construction "distorts" the original metric extensively. In this paper, we utilize the original metric on a metric space to construct a new metric. Under the new metric, the space is complete and uniformly locally almost convex. Although the new metric may not be topologically equivalent to the original, we give some conditions under which it is. We also give conditions for it to be almost convex, uniformly locally convex, or convex. The new metric is closely related to W. Wilson's "intrinsic metric" [8].

2. Definitions and terminology. For general concepts, we follow the notation of $[5]$. Let $(X, d)$ be a metric space. For each nonnegative integer $n$, set $u_{n}=\left\{(x, y) \in X \times X \mid d(x, y) \leqq 1 / 2^{n}\right\}$. The collection $\left\{u_{n}\right\}_{n=0}^{\infty}$ is a family of symmetric entourages satisfying the condition $u_{n} \circ u_{n} \subseteq u_{n-1}$ for each integer $n \geqq 1$. Letting $u^{k}$ denote $u \circ u \circ \cdots$ $\circ u$ ( $k$ times), we define $v_{k}=\bigcap\left\{u_{k+n}^{2^{n}} \mid n=0,1, \cdots\right\}$. Since $u_{n}^{2} \subseteq u_{n-1}$, finite induction yields that $u_{k+1}^{2^{n}} \subseteq u_{k+n-1}^{2^{n-1}} \subseteq \cdots \subseteq u_{k}$. That is, $v_{k}$ is a nested intersection. The collection $\left\{v_{k}\right\}_{k=0}^{\infty}$ is also a family of symmetric entourages satisfying $v_{k}^{2} \subseteq v_{k-1}$ for each positive integer. Gaal $[4$, p. 164] has proved a metrization theorem for such a sequence of entourages, giving a canonical construction for the metric. We give a slightly different form of that theorem here.

(2.1) THEOREM. Let $\left\{v_{k}\right\}_{k=0}^{\infty}$ be a sequence of symmetric entourages for $X$ such that $v_{k}^{2} \subseteq v_{k-1}$ for each positive integer $k$. Then there is a pseudometric e for $X$ such that

$$
\left\{(x, y) \mid e(x, y)<1 / 2^{n+1}\right\} \subseteq v_{n} \subseteq\left\{(x, y) \mid e(x, y) \leqq 1 / 2^{n}\right\} .
$$

Let $\Lambda$ denote infimum and $V$ supremum. The canonically constructed $e$ is defined by

$$
\begin{aligned}
& e(x, y)=1 \quad \text { if }(x, y) \notin u_{0}, \\
& e(x, y)=\wedge\left\{r \mid r=\sum_{i=1}^{n} 2^{-k_{i}} \text { and }(x, y) \in u_{k_{1}} \circ u_{k_{2}} \circ \cdots \circ u_{k_{n}}\right\} .
\end{aligned}
$$

Presented to the Society, August 30, 1968; received by the editors August 26, 1968. 
For the remainder of this paper, $X, d,\left\{u_{k}\right\},\left\{v_{k}\right\}$ and $e$ will be as above.

Let $(W, \rho)$ be a metric space and $f:[0,1] \rightarrow W$ a continuous function. The "curvelength" of $f$ is defined to be $V\left\{\sum_{i=0}^{k=0} \rho\left(f\left(x_{i}\right), f\left(x_{i+1}\right)\right)\right\}$ all possible finite partitions $0 \leqq x_{0}, x_{1} \leqq \cdots \leqq x_{n}=1$ of $\left.[0,1]\right\}$. Then the "arclength metric" on $X$ is defined by al $(x, y)=\Lambda$ \{curvelength of $f \mid$ all possible continuous $f:[0,1] \rightarrow(W, \rho)\}$. Note that the "arclength metric" may not be a metric since al $(x, y)=\infty$ is possible. If, however $\operatorname{al}(x, y)<\infty$ for all $(x, y) \in X \times X$, then it is a metric.

A metric space $(W, \rho)$ is (uniformly locally) convex if (there is an $\epsilon>0$ such that) given $x, y \in W$ and $\alpha \in[0,1]$, there is a $z \in W$ such that $\rho(x, z)+\rho(z, y)=\rho(x, y)$ and $\rho(x, z)=\alpha \rho(x, y)$ (whenever $\rho(x, y)$ $<\epsilon)$. It is almost convex if given $x, y \in W$ and $\delta>0$, there is a point $z \in W$ such that $\left|\frac{1}{2} \rho(x, y)-\rho(x, z)\right|<\delta$ and $\left|\frac{1}{2} \rho(x, y)-\rho(y, z)\right|<\delta$. Finally, $(W, \rho)$ is uniformly locally almost convex if there exists $\epsilon>0$ such that the $\epsilon$-neighborhood of each point is almost convex.

A uniformly locally compact space is one for which there exists $\delta>0$ such that the closed $\delta$-neighborhood of each point is compact. If all closed and bounded sets are compact, the space is called a Weierstrass space.

For an $\epsilon>0$, a metric space $(W, \rho)$ is $\epsilon$-chainable if given $u, v \in W$, there exists a sequence of points $u=u_{0}, u_{1}, \cdots, u_{n}=v$ (called an $\epsilon$-chain) such that $\rho\left(u_{i-1}, u_{i}\right) \leqq \epsilon$ for each $i=1,2, \cdots, n$. The length of the chain is $\sum_{i=1}^{n} \rho\left(u_{i-1}, u_{i}\right)$. The space is well-chained if it is $\epsilon$-chainable for any $\epsilon>0$. Note that none of the above properties are topological.

3. The space $(X, e)$. For any dyadic rational $r \in(0,1]$, define $r v=v_{k_{1}} \circ v_{k_{2}} \circ \cdots \circ v_{k_{n}}$, where $r=\sum_{i=1}^{n} 2^{-k_{i}}$ and $k_{i}<k_{i+1}$ for $i$ $=1,2, \cdots n-1$. Gaal $\left[4\right.$, p. 115] shows that ${ }_{r} v \circ_{8} v \subseteq_{r+8} v$ for any two dyadic rationals $r, s \in(0,1]$ such that $r+s \leqq 1$.

(3.1) Lemma. The collection of entourages $\left\{v_{k}\right\}$ satisfies $v_{\mathbf{k}+1}^{2}=v_{k}$ for each $k \geqq 0$.

Proof. It is sufficient to observe that

$$
\bigcap_{n=0}^{N} u_{k+1+n}^{2^{n}} \circ \bigcap_{n=0}^{N} u_{k+1+n}^{2^{n}}=\bigcap_{n=0}^{N+1} u_{k+n}^{2^{n}}
$$

is valid for each $N>0$. The nesting property then yields that

$$
\bigcap_{n=0}^{\infty} u_{k+1+n}^{2^{n}} \circ \bigcap_{n=0}^{\infty} u_{k+1+n}^{2^{n}}=\bigcap_{n=0}^{\infty} u_{k+n}^{2^{n}}
$$


(3.2) CorollaRy. For each dyadic rational $r \in(0,1 / 2),{ }_{r} v \circ{ }_{r} v={ }_{2 r} v$.

(3.3) Theorem. The space $(X, e)$ is uniformly locally almost convex.

Proof. Choose $\epsilon=1$. Let $\delta>0$ and points $x, y \in X$ with $e(x, y)<1$ be given. Select a dyadic rational $n / 2^{k}<1$ such that $0<n / 2^{k}-e(x, y)$ $<\delta$. Since $e(x, y)<n / 2^{k},(x, y) \in v_{k}^{n} \subseteq v_{k+1}^{2 n}$. Thus there is a sequence $x=x_{0}, x_{1}, \cdots, x_{2 n}=y$ such that $\left(x_{i}, x_{i+1}\right) \in v_{k+1}$ for each $i$. We will show that $\left|2 e\left(x, x_{n}\right)-e(x, y)\right|<\delta$. The symmetry will then yield the desired result.

Since $\left(x, x_{n}\right) \in v_{k+1}^{n}, e(x, y)<n / 2^{k+1}$. We have

$$
\begin{aligned}
0 & <n / 2^{k}-2 e\left(x, x_{n}\right) \\
& =\left(n / 2^{k}-e(x, y)\right)+e(x, y)-2 e\left(x, x_{n}\right) \\
& <\delta+e(x, y)-2 e\left(x, x_{n}\right),
\end{aligned}
$$

so that $2 e\left(x, x_{n}\right)-e(x, y)<\delta$. On the other hand, $e(x, y) \leqq e\left(x, x_{n}\right)$ $+e\left(x_{n}, y\right)$. Now $e\left(x_{n}, y\right)<n / 2^{k+1}$, so

$$
2 e(x, y)-2 e\left(x, x_{n}\right) \leqq 2 e\left(x_{n}, y\right) \leqq n / 2^{k} \leqq e(x, y)+\delta .
$$

Thus $e(x, y)-2 e\left(x, x_{n}\right) \leqq \delta$. This, together with the above inequality, establishes the desired result.

(3.4) CoRollary. If $\left\{w_{k}\right\}_{k=0}^{\infty}$ is a sequence of symmetric entourages for a space $W$ such that $w_{k}^{2}=w_{k-1}$ for each positive integer $k$, then the pseudometric $\rho$ constructed by (2.3) is uniformly locally almost convex.

If $(X, e)$ is $\epsilon$-chainable space for some $\epsilon<1$, a new metric can be defined for $X$ as follows. For each positive dyadic rational $r=\sum_{i=1}^{n} 2^{-k_{i}}$, set ${ }_{r} v=v_{k_{1}} \circ v_{k_{2}} \circ \cdots \circ v_{k_{n}}$. Since $v_{k+1}^{2}=v_{k}$, this definition of $r v$ is welldefined. Define $e^{*}(x, y)=\Lambda\left\{r \mid(x, y) \in_{r} v\right\}$. Since $(X, e)$ is $\epsilon$-chainable, $(x, y)$ is in $r v$ for a sufficiently large $r$. Furthermore, $e^{*}(x, y)=e(x, y)$ if $e(x, y)<\epsilon$. Symmetry and the triangular inequality are "built in" the definition, and are trivial to verify. The metric $e^{*}$ is analogous to the "intrinsic metric" of W. Wilson [8].

(3.5) Theorem. Let $(X, e)$ be $\epsilon$-chainable for some $\epsilon<1$. Then $\left(X, e^{*}\right)$ is an almost convex space.

The proof is exactly the same as that of the previous theorem, except that the points $x, y \in X$ can be chosen arbitrarily.

(3.6) Proposition. Let $(W, \rho)$ be well-chained and uniformly locally compact. Then $W$ has a metric $\rho^{*}$ such that $\left(W, \rho^{*}\right)$ is a Weierstrass space and $\rho=\rho *$ locally. 
Proof. Let $\delta$ be such that the closed $2 \delta$-neighborhood of each point is compact. Define

$$
\rho^{*}(x, y)=\Lambda\left\{\sum \rho\left(x_{i-1}, x_{i}\right) \mid x=x_{0}, x_{1}, \cdots, x_{n}=y \text { is a } \delta \text {-chain }\right\} .
$$

Clearly $\rho=\rho^{*}$ locally. The triangular inequality and symmetry are built into $\rho^{*}$.

Let $A$ be any closed and bounded set, and $a \in A$. (Trivially, $A=\varnothing$ is compact.) Since $A$ is bounded, for some $k>0, \operatorname{diam}(A)$ (=diameter of $A)<k \delta$. Then, setting $w_{\delta}=\{(x, y) \mid \rho(x, y) \leqq \delta\}$, we have $w_{\delta}^{k}(a)$ is compact [5, p. 214]. But $A \subseteq w_{\delta}^{k}(a)$, so $A$ is compact.

(3.7) Theorem. If $(X, e)$ is uniformly locally compact, then $(X, e)$ is uniformly locally convex. If $(X, e)$ is also $\epsilon$-connected for some $\epsilon<1$, then $\left(X, e^{*}\right)$ is convex.

Proof. Let $\epsilon$ be such that each closed set of diameter less than $\epsilon$ is compact. Then for a closed set $A$ with diameter less than $\epsilon \wedge 1 / 2, A$ is almost convex and compact. It is well known that almost convexity and compactness imply convexity.

For the remainder, it is sufficient to observe that the metric $e^{*}$ is constructed analogously to $\rho^{*}$. Hence $\left(X, e^{*}\right)$ is a Weierstrass space. Wilson [8] proves, but does not state explicitly that an almost convex Weierstrass space is convex.

The last theorem cannot be sharpened very much, as is easily seen from the following example.

(3.8) ExAmple. Let $U=\left\{(x, y) \in R^{2} \mid\right.$ for some integer $n>0, y$ $=\exp (n \log x /(n+1)), 0 \leqq x \leqq 1\}$. If $U$ is endowed with the arclength metric, then $U$ is almost convex and complete. It is not convex, since $\rho((0,0),(1,1))=\sqrt{ } 2$, but there is no point $\left(x_{0}, y_{0}\right)$ such that $\rho\left((0,0),\left(x_{0}, y_{0}\right)\right)=\rho\left(\left(x_{0}, y_{0}\right),(1,1)\right)=\sqrt{ } 2 / 2$. Furthermore, the metric $\sigma$ constructed from $\rho$ as in the introduction coincides with $\rho$ for distances less than $1 / 2$. Hence, the extension $\sigma^{*}$ coincides in all cases with $\rho$.

Generally speaking, the properties of $(X, d)$ are not carried over to $(X, e)$. For instance, let $X=[0,1]$ and $d(x, y)=|x-y|^{1 / 2}$. Since $(X, d)$ is a Peano continuum, it is compact, connected, and separable. None of these properties carry over to $(X, e)$, since $e(x, y)=1$ if $x \neq y$ and $e(x, x)=0$. However, we do have the following rather startling result, which is an extension of a theorem due to S. B. Myers [7].

(3.9) Theorem. Let $(X, d)$ be complete. Then $(X, e)$ is complete.

Proof. Let $x_{n}$ be a Cauchy sequence under $e$. Since $d(x, y) \leqq e(x, y)$ 
for all $x, y \in X, x_{n}$ is a Cauchy sequence under $d$. Let $x_{n}$ converge to $x_{0}$ under $d$. We will show that $x_{n}$ converges to $x_{0}$ under $e$.

Without loss of generality, we may assume that the sequence $x_{n}$ satisfies (a) $e\left(x_{m}, x_{n}\right) \leqq 1 / 2^{N}$ if $n, m \geqq N$, and (b) $d\left(x_{n}, x_{0}\right) \leqq 1 / 2^{n}$. Let $K>0$ be given. We will show that for $n>K+1, e\left(x_{n}, x_{0}\right) \leqq 1 / 2^{K}$. To do this, it is sufficient to show that $\left(x_{n}, x_{0}\right) \in v_{K}$ for $n>K$. Since $v_{K}=\bigcap_{j=0}^{\infty} u_{K+j}$, we need only show that, given $j$, we can find a chain of points $x_{n}=y_{0}, y_{1}, y_{2}, \cdots, y_{m}=x_{0}$ such that $\left(y_{i-1} y_{i}\right)_{a} \in u_{K+j}$ for each $i$ and $m \leqq 2 j$. For the given $j>0$, choose as the point $y_{m-1}$ the point $x_{K+j}$ from the Cauchy sequence. Since $n$ and $K+j$ are greater than $K, e\left(x_{n}, x_{K+j}\right) \leqq 1 / 2^{K+1}$. That is $\left(x_{n}, x_{K+j}\right) \in v_{K+1}=\bigcap_{i=1}^{\infty} u_{K+1+i}^{2^{i}} \subseteq u_{K+1}$. Hence there exists a sequence of points $x_{n}=y_{0}, y_{1}, \cdots, y_{2^{i-1}}=x_{K+j}$ such that $\left(x_{i-1}, x_{i}\right) \in u_{K+j}$. Thus setting $m=2^{j-1}+1 \leqq 2^{j}$ we have the desired sequence.

The author was unable to find the following result in the literature, although it is probably well-known.

(3.10) THEOREM. If $(W, \rho)$ is a complete almost convex metric space, then $(W, \rho)$ is arcwise connected and the "arclength metric" is equal to $\rho$.

Proof. We will prove both parts at once as follows. Since $\rho(x, y)$ $\leqq \mathrm{al}(x, y)$ under all circumstances, we need show that only for $m>0$, can an arc be constructed in $W$ whose length is less than $\rho(x, y)+2^{-m}$. The technique used is related to that used by Menger [6] in his classic paper on convex metrics.

We define a function $f:[0,1] \rightarrow W$ as follows. Let $f(0)=x$ and $f(1)=y$. Then let $f(1 / 2)$ be a point in $W$ such that $\mid 2 \rho(f(0), f(1 / 2))$ $-\rho(f(0), f(1)) \mid<1 / 2^{m+2} \cdot 1 / 2$ and $|2 \rho(f(1), f(1 / 2))-\rho(f(0), f(1))|$ $<1 / 2^{m+2} \cdot 1 / 2$.

Assume now that we have chosen points $f\left(0 / 2^{k}\right), f\left(1 / 2^{k}\right), \cdots, f(1)$, satisfying

(a) $\left|2 \rho\left(f\left(\frac{2 n}{2^{k}}\right), f\left(\frac{2 n+1}{2^{k}}\right)\right)-\rho\left(f\left(\frac{2 n}{2^{k}}\right), f\left(\frac{2 n+2}{2^{k}}\right)\right)\right|$

$<1 / 2^{m+2} \cdot 1 / 2^{k}$,

(b) $\left|2 \rho\left(f\left(\frac{2 n+2}{2^{k}}\right), f\left(\frac{2 n+1}{2^{k}}\right)\right)-\rho\left(f\left(\frac{2 n}{2^{k}}\right), f\left(\frac{2 n+2}{2^{k}}\right)\right)\right|$

$<1 / 2^{m+2} \cdot 1 / 2^{k}$.

We then choose for each successive pair of points, $f\left(n / 2^{k}\right)$ and $f\left(n+1 / 2^{k}\right)$, a point $f\left(2 n+1 / 2^{k+1}\right)$ such that (a) and (b) are satisfied. 
This inductive process defines $f$ on the dyadic rationals of $[0,1]$. Now note that

$$
\begin{gathered}
\rho\left(f\left(\frac{2 n+1}{2^{k}}\right), f\left(\frac{2 n+2}{2^{k}}\right)\right) \leqq \frac{1}{2^{m+2}} \frac{1}{2^{k+1}} \\
+1 \frac{1}{2} \rho\left(f\left(\frac{2 n}{2^{k}}\right), f\left(\frac{2 n+2}{2^{k}}\right)\right)
\end{gathered}
$$

and

$$
\rho\left(f\left(\frac{2 n}{2^{k}}\right), f\left(\frac{2 n+1}{2^{k}}\right)\right) \leqq \frac{1}{2^{m+2}} \cdot \frac{1}{2^{k+1}}+\frac{1}{2} \rho\left(f\left(\frac{2 n}{2^{k}}\right), f\left(\frac{2 n+2}{2^{k}}\right)\right)
$$

holds for all $n$. Thus we have

$$
\rho(f(1 / 2), f(0)) \leqq \frac{1}{2}\left(\frac{1}{2^{m+2}}+\rho(f(0), f(1))\right)
$$

and

$$
\rho(f(1 / 2), f(1)) \leqq \frac{1}{2}\left(\frac{1}{2^{m+2}}+\rho(f(0), f(1))\right) .
$$

Induction then shows that

$$
\rho\left(f\left(\frac{2 n+1}{2^{k}}\right), f\left(\frac{2 n}{2^{k}}\right)\right) \leqq \frac{1}{2^{k}}\left(\frac{1}{2^{m+1}}+\rho(f(0), f(1))\right)
$$

and

$$
\rho\left(f\left(\frac{2 n+1}{2^{k}}\right), f\left(\frac{2 n+2}{2^{k}}\right)\right) \leqq \frac{1}{2^{k}}\left(\frac{1}{2^{m+1}}+\rho(f(0), f(1))\right) .
$$

Thus $f$ is uniformly continuous-in fact, it satisfies a Lipschitz condition with constant $1 / 2^{m+1}+\rho(f(0), f(1))$. We extend $f$ to $[0,1]$, maintaining the Lipschitz condition. Since the Lipschitz constant is $1 / 2^{m+1}+\rho(f(0), f(1))$, the length of the curve is $\leqq 1 / 2^{m+1}+\rho(f(0), f(1))$ [2].

(3.11) Corollary. The space $(X, e)$ is uniformly locally arcwise connected, and locally, the "arclength metric" on $(X, e)$ is equal to $e$.

We conclude with some remarks on uniform equivalence of $(X, d)$ and $(X, e)$. Since $v_{n} \subseteq u_{n}$ for all $n$, it is clear that the " $e$ " uniformity contains the " $d$ " uniformity. If for some $n$ and some $k, u_{n} \subseteq v_{k}$, then the " $d$ " uniformity also contains the " $e$ " uniformity. That is, 
(3.12) Theorem. The spaces $(X, d)$ and $(X, \rho)$ are uniformly equivalent iff there exist $k$ and $n$ such that $u_{n} \subseteq v_{k}$.

Two metric spaces are called Lipschitz equivalent if there is a function from one to the other such that both the function and its inverse satisfy a local Lipschitz condition.

(3.13) Theorem. The spaces $(X, d)$ and $(X, e)$ are Lipschitz equivalent iff there exists $n>0$ such that $u_{n+k} \subseteq v_{k}$ for all $k$.

Proof. This is essentially a corollary to a theorem in [3], which characterizes Lipschitz equivalence in terms of entourage sequences.

\section{BIBLIOGRAPHY}

1. R. H. Bing, Partitioning a set, Bull. Amer. Math. Soc. 55 (1949), 1101-1110.

2. R. Fraser, On Lipschitz functions, Ph.D. Thesis, Rutgers, New Brunswick, N. J., 1967.

3. - Axiom systems for Lipschitz structures, Fund. Math. (to appear).

4. S. A. Gaal, Point set topology, Academic Press, New York, 1964.

5. J. L. Kelley, General topology, Van Nostrand, Princeton, N. J., 1955.

6. K. Menger, Üntersuchungen über allgemeine Metrik, Math. Ann. 100 (1928), 75-163.

7. S. B. Myers, Arcs and geodesics in metric spaces, Trans. Amer. Math. Soc. $\mathbf{5 7}$ (1945), 217-227.

8. W. A. Wilson, On rectifiability in metric spaces, Bull. Amer. Math. Soc. 38 (1932) 419-426.

Louisiana State University 